\title{
Effect of compositions in nanostructured lipid carriers (NLC) on skin hydration and occlusion
}

\author{
This article was published in the following Dove Press journal: \\ International Journal of Nanomedicine \\ 21 December 2012 \\ Number of times this article has been viewed
}

\section{$\mathrm{CH} \mathrm{Loo}^{1,2}$ \\ $M$ Basri $^{2}$ \\ R Ismail' \\ HLN Lau' \\ BA Tejo ${ }^{2}$ \\ MS Kanthimathi ${ }^{3}$ \\ HA Hassan' \\ YM Choo'}

'Malaysian Palm Oil Board, Bandar Baru Bangi, ${ }^{2}$ Department of Chemistry, Universiti Putra Malaysia, Serdang, ${ }^{3}$ Department of Molecular Medicine, University of Malaya, Kuala Lumpur, Malaysia
Correspondence: Mahiran Basri Department of Chemistry, University Putra, 43400 UPM Serdang, Selangor Darul Ehsan, Malaysia Tel +60389255708

Fax +60389256197

Email mahiran@science.upm.edu.my
Purpose: To study the effects of varying lipid concentrations, lipid and oil ratio, and the addition of propylene glycol and lecithin on the long-term physical stability of nanostructured lipid nanocarriers (NLC), skin hydration, and transepidermal water loss.

Methods: The various NLC formulations (A1-A5) were prepared and their particle size, zeta potential, viscosity, and stability were analyzed. The formulations were applied on the forearms of the 20 female volunteers (one forearm of each volunteer was left untreated as a control). The subjects stayed for 30 minutes in a conditioned room with their forearms uncovered to let the skin adapt to the temperature $\left(22^{\circ} \mathrm{C} \pm 2{ }^{\circ} \mathrm{C}\right)$ and humidity $(50 \% \pm 2 \%)$ of the room. Skin hydration and skin occlusion were recorded at day one (before treatment) and day seven (after treatment). Three measurements for skin hydration and skin occlusion were performed in each testing area.

Results: NLC formulations with the highest lipid concentration, highest solid lipid concentration, and additional propylene glycol (formulations A1, A2, and A5) showed higher physical stability than other formulations. The addition of propylene glycol into an NLC system helped to reduce the particle size of the NLC and enhanced its long-term physical stability. All the NLC formulations were found to significantly increase skin hydration compared to the untreated controls within 7 days. All NLC formulations exhibited occlusive properties as they reduced the transepidermal water loss within 7 days. This effect was more pronounced with the addition of propylene glycol or lecithin into an NLC formulation, whereby at least $60 \%$ reduction in transepidermal water loss was observed.

Conclusion: NLCs with high lipid content, solid lipid content, phospholipid, and lecithin are a highly effective cosmetic delivery system for cosmetic topical applications that are designed to boost skin hydration.

Keywords: nanostructured lipid carriers, transepidermal water loss, skin hydration, particle size

\section{Introduction}

The stratum corneum consists of corneocytes filled with keratin and embedded in a lipid matrix. Regulation of the penetration of a topically applied product and hydration across the stratum corneum depends on this lipid rich matrix. ${ }^{1-3}$ Lipids mainly found in the stratum corneum are phospholipids, cholesterol-3-sulphate, cholesterol, ceramides, sterol esters, and free fatty acids. Sebaceous lipids found in the stratum corneum consist of triglycerides, wax esters, and squalene. The organized structure of lipids is related to the barrier properties of the skin., ${ }^{4,5}$

The skin is a viscoelastic organ. ${ }^{6,7}$ Elastic components are made of collagen and elastic fibers; viscous parts are formed based on friction between cells and collagen 
bundles and other components of the dermal matrix. ${ }^{8}$ The stratum corneum normally maintains a $10 \%-20 \%$ hydration state. ${ }^{9}$ Water and lipid content strongly influences the frictional resistance of the skin. ${ }^{10}$ Perturbation of water content in the skin might cause functional defect and clinical symptoms. Therefore, skin hydration and occlusion are of primary interest in the cosmetic and dermatology fields.

Transepidermal water loss is a good indicator of impaired barrier function of the stratum corneum. An increase in transepidermal water loss indicates a disruption of the stratum corneum and loss of intercellular lipids. Skin hydration is measured by capacitance or conductance and demonstrates acute, chronic, narrow, or wide oscillations in a variety of skin conditions. ${ }^{11}$ Skin that is properly hydrated has been known to age more slowly than moisture-deprived skin and is better protected against environmental influences. An increase in skin hydration and lipid content helps to improve viscous resistance against deformation. ${ }^{12}$

Most topical preparations have an undesirable esthetic appearance and feel which is unacceptable to consumers (eg, petrolatum); therefore, the demand for novel occlusive agents or lipids is rising. Nanostructured lipid carriers (NLCs) consist of a solid lipid matrix with a certain amount of liquid lipid, and were introduced as a novel delivery system for various cosmetic active ingredients and pharmaceutical drugs. ${ }^{13}$ They have great appeal in the cosmetic field as they are able to enhance the chemical stability of actives, provide occlusive effect to the skin and therefore increase skin hydration, enhance the skin bioavailability of actives, and increase the physical stability of topical formulations. $^{14}$

NLCs could provide better skin hydration and occlusion compared to traditional delivery systems (eg, macroemulsion) due to its ultrafine size. NLCs localized on the skin leads to a formation of film. This film has an occlusive action to prevent water loss from the skin through evaporation. The degree of occlusion skin, is based on the size of the particles. The smaller the size of the particles, the less water that evaporates from the skin whereas the bigger the size of the particles, the more water that evaporates from the skin. ${ }^{14}$

Occlusion and skin hydration are influenced by the delivery system or vehicle and the ingredients used in a formulation. Limited studies related to the effect of the NLC delivery system on skin hydration and occlusion have been published. No study was found that explains the effect of various ingredients incorporated in the formulation on skin hydration and occlusion.

\section{Material and methods}

\section{Materials}

Span $^{\text {TM }} 40$ (sorbitan monopalmitate) and Tween ${ }^{\circledR} 80$ (polysorbate 80) were obtained from Croda International (Goole, United Kingdom). Isopropyl palmitate was purchased from InterMed Sdn Bhd (Kuala Lumpur, Malaysia). Lipocire $^{\mathrm{TM}}$ DM (hydrogenated palm kernel glycerides) was purchased from Gattefosse (Lyon, France). Concentrated palm phytonutrients (major components are tocols and carotenoids) were obtained from the Malaysian Palm Oil Board (Kuala Lumpur, Malaysia). Propylene glycol and Phospholipon ${ }^{\circledR} 85 \mathrm{G}$ (soy lecithin enriched with $85 \%$ phosphatidylcholine) was purchased from Lipoid GmbH (Ludwigshafen, Germany).

\section{Preparation of nanostructured lipid carrier (NLC)}

Melted lipid phase containing solid lipid (hydrogenated palm kernel glycerides), liquid lipid (isopropyl palmitate), $3.00 \%$ lipophilic surfactant (Span 40 ) and $0.40 \%$ bioactive compounds (palm-based phytonutrients concentrates) were added to hot aqueous solution containing 3.00\% hydrophilic surfactant (Tween 80). Both phases (lipid and water phases) were heated to $70^{\circ} \mathrm{C}$. The mixture was homogenized by Polytron $^{\circledR}$ PT 3100 homogenizer (Kinematica AG, Luzern, Switzerland) at 10,000 rpm for 2 minutes. The macroemulsion formed was cooled in an ice bath to room temperature $\left(25^{\circ} \mathrm{C}\right)$. The pre-emulsion was further homogenized at $85^{\circ} \mathrm{C}$ using a high pressure homogenizer for three cycles at 750 bars. The lipid dispersion was then cooled in an ice bath to room temperature $\left(25^{\circ} \mathrm{C}\right) .{ }^{15}$ Compositions of the NLC formulations are shown in Table 1.

Table I Compositions of the nanostructured lipid carrier formulations

\begin{tabular}{|c|c|c|c|c|c|}
\hline \multirow[t]{2}{*}{ Ingredient } & \multicolumn{5}{|c|}{ Formulation (\%, weight/weight) } \\
\hline & AI & A2 & A3 & A4 & A5 \\
\hline Span ${ }^{\mathrm{TM}} 40$ & 3.00 & 3.00 & 3.00 & 3.00 & 3.00 \\
\hline Tween $^{\circledR} 80$ & 3.00 & 3.00 & 3.00 & 3.00 & 3.00 \\
\hline Lipocire $^{\mathrm{TM}} \mathrm{DM}$ & 17.64 & 26.64 & 9.80 & 17.64 & 17.64 \\
\hline IPP & 1.96 & 2.96 & 9.80 & 1.96 & 1.96 \\
\hline CPP & 0.40 & 0.40 & 0.40 & 0.40 & 0.40 \\
\hline Phenonip ${ }^{\circledR}$ & 0.70 & 0.70 & 0.70 & 0.70 & 0.70 \\
\hline Lecithin & - & - & - & I & - \\
\hline Propylene glycol & - & - & - & - & 2.00 \\
\hline Water & qs 100 & qs 100 & qs 100 & qs 100 & qs 100 \\
\hline
\end{tabular}

Abbreviations: AI, nanostructured lipid carrier with $20 \%$ lipids and a solid lipid/liquid lipid ratio of 90:10; A2, nanostructured lipid carrier with 30\% lipids; A3, nanostructured lipid carrier with $20 \%$ lipids and a solid lipid/liquid lipid ratio of 50:50; A4, nanostructured lipid carrier with lecithin added; A5, nanostructured lipid carrier with propylene glycol added; CPP, concentrated palm phytonutrients; IPP, isopropyl palmitate; qs, quantum satis. 


\section{Particle size analysis}

Particle size analysis for NLC was performed using photon correlation spectroscopy with a high performance particle sizer (HPP5001; Malvern Instruments Ltd, Malvern, United Kingdom). In photon correlation spectroscopy, the intensity fluctuations of scattered light arising from Brownian motion are measured. The size distribution of the particles was measured by the Stokes-Einstein equation. The mean particle size was obtained from the average of five measurements (ten 30-second runs) at an angle of 90 degrees. The volume of the sample required was $10 \mathrm{~mm}$. All samples were diluted in distilled water to weak opalescence. The dispersant (water) had a refractive index of 1.333 and viscosity of $0.8905 \mathrm{cP}$ at $25^{\circ} \mathrm{C}$.

\section{Zeta potential analysis}

Zeta potential reflects the electric charge on the particle surface and physical stability of colloidal systems. ${ }^{16}$ This can be determined by the electrophoretic mobility of particles and colloids dispersed in a liquid. The zeta potential analysis was performed using a Zetasizer ${ }^{\circledR}$ Nano Z (ZEN 2600; Malvern Instruments) with five measurements (ten runs). The samples were diluted with distilled water and added into the equipment chamber. Particles with a large negative or positive zeta potential (more negative than $-30 \mathrm{mV}$ or more positive than $+30 \mathrm{mV}$ ) will repel each other and will not aggregate. ${ }^{17}$

\section{Rheological measurements}

The viscosity of samples at $10 \mathrm{~s}^{-1}$ was recorded. Oscillatory stress sweep analysis was carried out between $0.1-100.0 \mathrm{~Pa}$ using a Physica MCR 300 rheometer (Anton Paar GmbH, Graz, Austria). The geometry of the cone-and-plate used in this test was CP 50-1. ${ }^{18}$ Dynamic oscillation testing is used to investigate the microscopic structures of viscoelastic materials, which provides information on the viscosity and elastic properties of the investigated delivery systems. ${ }^{19}$ In the stress sweep test, the oscillation frequency was fixed at $1 \mathrm{~Hz}$ in order to obtain a linear viscoelastic (LVE) region. The region where $\mathrm{G}^{\prime}$ (the storage modulus) is independent of applied shear stress up to the critical strain value $\left(\gamma_{c}\right)$ is known as the LVE region. ${ }^{20}$

\section{Accelerated stability testing}

The stability of the NLCs was investigated by subjecting the formulation at room temperature $\left(25^{\circ} \mathrm{C}\right), 45^{\circ} \mathrm{C}$, and $5^{\circ} \mathrm{C}$ for 3 months. The NLCs were also centrifuged at $3500 \mathrm{rpm}$ for 15 minutes. Three freeze-thaw cycles (one cycle $=$ first day at $5^{\circ} \mathrm{C}$ and second day at $25^{\circ} \mathrm{C}$ ) were performed to investigate the stability of the NLCs. The phase separation of NLCs was observed under polarized light. ${ }^{15}$

\section{Efficacy testing (skin hydration and transepidermal water loss)}

Twenty Asian female volunteers with healthy skin participated in this study. All the volunteers were older than 40 years. All the volunteers participating in the study gave their informed written consent. The protocol, informed consent agreement, and any protocol amendments were reviewed and approved by an institutional review board. Each volunteer was instructed not to wash or use any other product on her forearm for at least 5 hours before the test. The evaluation was carried out on the forearm, in an area of $4 \mathrm{~cm}^{2}$. Two areas of $4 \mathrm{~cm}^{2}$ were marked on the forearm: one was treated with the product while the other was kept untreated as a control. The formulations were applied on the forearms of the volunteers. The subjects stayed for 30 minutes in a conditioned room with their forearms uncovered to let the skin adapt to the temperature $\left(22^{\circ} \mathrm{C} \pm 2^{\circ} \mathrm{C}\right)$ and humidity $(50 \% \pm 2 \%)$ of the room. Skin hydration and skin occlusion were recorded at day one (before treatment) and day seven (after treatment). Three measurements for skin hydration and skin occlusion were performed in each testing area.

The effect of the samples on the skin hydration was evaluated using a Corneometer ${ }^{\circledR}$ CM 825 (Courage + Khazaka Electronic GmbH, Cologne, Germany). The measuring principle of the instrument is based on the capacitance measurement of a dielectric medium. The dielectric constant of the skin changes with the water content. The changes in water content of the stratum corneum are converted to arbitrary units of hydration. Transepidermal water loss from the skin was investigated using Tewameter ${ }^{\mathrm{B} T M} 300$ (Courage + Khazaka). The principle of this test is based on the diffusion principle in an open chamber. The results are given in arbitrary units and expressed as an average percentage change of skin hydration and skin occlusion due to the application of a sample at each measurement point. ${ }^{12}$ All data are expressed as mean \pm standard deviation and were statistically analyzed by one-way analysis of variance. Differences between the samples and the untreated control group were evaluated by Tukey's $t$-test. $P<0.05$ was considered significant.

\section{Results and discussion}

\section{Particle size analysis}

To investigate the influence of lipid concentration, NLCs (formulation A1 and A2) composed of $20 \%$ and $30 \%$ lipid phase (solid lipid/liquid lipid ratio of 90:10) were prepared. 
Particle size of formulation A2 had a slightly bigger particle size $(218.60 \pm 5.76 \mathrm{~nm})$ when compared to formulation A1 (193.00 $\pm 1.33 \mathrm{~nm}$ ) (Figure 1). The homogenization energy exerted for both NLCs was the same, but mass of disperse phase was increased from $20 \%$ to $30 \%$; therefore, less dispersion energy was available per unit lipid leading to bigger particle size. This result is in agreement with the findings of Teeranachaideekul et al and Phipps in which the higher the lipid content, the higher the mean particle size obtained. $^{21,22}$

Particle size of formulation A1 remained almost unchanged for 3 months, indicating that A1 was stable at room temperature within the tested time (Figure 1). Particle size for A2 increased from the first $(219.60 \pm 5.76 \mathrm{~nm})$ to the third month $(273.40 \pm 3.92 \mathrm{~nm})$ (Figure 1). This finding is in agreement with the findings from Seetapan et al in which NLCs with high lipid concentration were physically less stable compared to NLCs with low lipid concentration due to their ability to more easily recrystallize. ${ }^{23}$

The physical stability of the NLC with high solid lipid content (solid lipid/liquid lipid ratio of 90:10; formulation A1) was higher when compared to the NLC with low solid lipid content (solid lipid/liquid lipid ratio of 50:50; formulation A3). The particle size of formulation A1 remained almost unchanged in 3 months, whereas the particle size of A3 increased 19\% from the first to the second month (Figure 1). This could be due to the presence of large quantities of solid lipid, which reduced the coalescence of droplets. $^{24}$

The NLC with lecithin (formulation A4) showed bigger particle size $(225.40 \pm 1.84 \mathrm{~nm})$ compared to the NLC without lecithin (formulation A1; $193 \pm 1.33 \mathrm{~nm}$ ) and exhibited

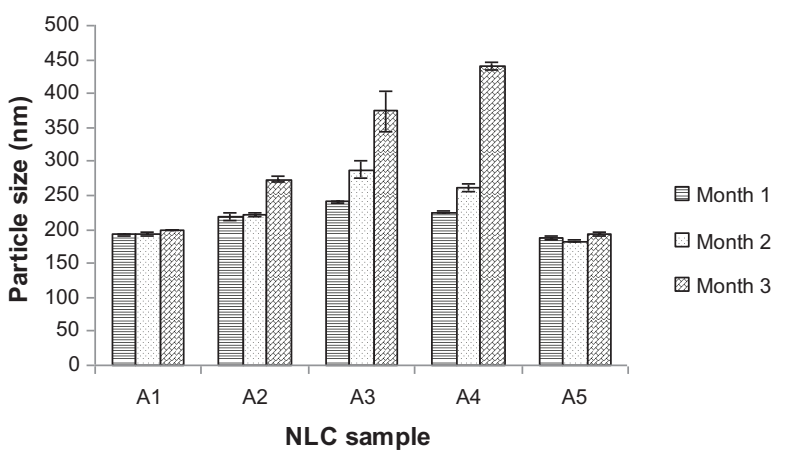

Figure I Particle size of the nanostructured lipid carrier formulations during 3-month storage.

Abbreviations: AI, nanostructured lipid carrier with 20\% lipids and a solid lipid/liquid lipid ratio of 90:10; A2, nanostructured lipid carrier with $30 \%$ lipids; A3, nanostructured lipid carrier with $20 \%$ lipids and a solid lipid/liquid lipid ratio of 50:50; A4, nanostructured lipid carrier with lecithin added; A5, nanostructured lipid carrier with propylene glycol added; NLC, nanostructured lipid carrier. lower physical stability as its particle size increased from the first month to the third month (Figure 1). This could be due to the emulsification process which could produce an unstable system consisting of micelles and lamellar aggregates in the presence of lecithin. This kind of system suffers a dynamic process of lecithin exchange between the particles, which leads to phase separation and structural modification. ${ }^{25}$

The particle size of the NLC with propylene glycol (formulation A5; $188.40 \pm 2.72 \mathrm{~nm}$ ) was slightly smaller when compared to the NLC without propylene glycol (formulation A1; $193 \pm 1.33 \mathrm{~nm}$ ) (Figure 1). Formulation A5 was physically stable as its particle size remained unchanged from the first to the third month (Figure 1). This result is in agreement with the findings from Chanana and Sheth in which the addition of propylene glycol in an emulsion system helps to reduce particle size and enhance physical stability. ${ }^{26}$ They found that propylene glycol was more effective in reducing the particle size of an emulsion compared to glycerol. Addition of polyhydric alcohol to an emulsion was found to increase the viscosity of the emulsion, which thus enhanced the physical stability of the emulsion.

\section{Zeta potential analysis}

Zeta potential was used to measure the particle charge and electrostatic repulsion. It is the potential at the surface of shear and it is measured in millivolts. Zeta potential is usually used to determine the stability of emulsion system. According to the Derjaguin-Landau-Verwey-Overbeek theory, ${ }^{16}$ a system could be classified as stable if the electrostatic repulsion dominated the attractive Van der Waals forces. Particles with zeta potentials more positive than $+30 \mathrm{mV}$ and more negative than $-30 \mathrm{mV}$ are normally considered stable. ${ }^{27}$ If the particles have a large negative or positive zeta potential, they will repel each other and thus are more stable.

The zeta potentials of A1, A2, A3, A4, and A5 ranged from $-31 \mathrm{mV}$ to $-36 \mathrm{mV}$, suggesting acceptable electrostatic stability of the NLC formulations (Table 2). The results did not correlate with the results obtained from particle size analysis, stress sweep test, and accelerated stability testing. The zeta potential results showed that formulations A3 and A4 were physically stable, whereas the results of particle size, accelerated stability testing, and stress sweep showed that formulations A3 and A4 were the most unstable when compared with other formulations. These observations are in agreement with the findings of Lippacher et al in which the results obtained from rheological, morphological, and differential scanning calorimetry analyses did not correlate with zeta potential analysis in predicting the physical stability 
Table 2 Physical characteristics of nanostructured lipid carrier formulations

\begin{tabular}{llll}
\hline Sample & Zeta potential $(\mathbf{m V})$ & Viscosity $(\mathbf{c P})$ & LVE $(\mathbf{P a})$ \\
\hline AI & $-32.57 \pm 0.26$ & $27.03 \pm 3.60$ & 0.32 \\
A2 & $-31.15 \pm 0.53$ & $40.12 \pm 4.33$ & 2.00 \\
A3 & $-33.17 \pm$ & $10.18 \pm 0.11$ & - \\
A4 & $-35.57 \pm$ & $46.66 \pm 3.60$ & 0.21 \\
A5 & $-31.23 \pm$ & $32.28 \pm 2.74$ & 0.51 \\
\hline
\end{tabular}

Abbreviations: AI, nanostructured lipid carrier with $20 \%$ lipids and a solid lipid/liquid lipid ratio of 90:10; A2, nanostructured lipid carrier with 30\% lipids; A3, nanostructured lipid carrier with $20 \%$ lipids and a solid lipid/liquid lipid ratio of 50:50; A4, nanostructured lipid carrier with lecithin added; A5, nanostructured lipid carrier with propylene glycol added; LVE, linear viscoelastic region.

of the loaded solid lipid nanoparticles. ${ }^{19}$ This could be due to the determination of zeta potential being carried out in a diluted state, whereby the interparticle interaction between particles is negligible. Therefore it cannot be used to explain the real condition of the prepared semisolid dispersions.

\section{Rheological measurement Viscosity}

Table 2 shows that formulation A2 $(40.12 \pm 4.33 \mathrm{cP})$ had higher viscosity when compared to formulation A1 $(27.03 \pm 3.60 \mathrm{cP})$. The increase in viscosity could be due to an increase in the volume of lipid phase from $20 \%$ to $30 \%$. These findings were in agreement with the findings of Floyd, which suggested that viscosity was increased with increasing lipid concentration. ${ }^{28}$ Formulation A3 had lower viscosity when compared to formulation $\mathrm{A} 1$ due to the lower solid lipid content in A3 (Table 2). These results were in agreement with the findings of Freitas and Muller, in which increased solid lipid content led to an increase in viscosity accompanied by a lack of lattice defects within lipid particles. ${ }^{29}$

The viscosity of formulation A4 $(46.66 \pm 3.60 \mathrm{cP})$ was higher than formulation $\mathrm{A} 1(27.03 \pm 3.60 \mathrm{cP})$ due to the presence of lecithin (Table 2). These findings were in agreement with the findings of Ishii et al in which the viscosity of soy bean oil emulsions stabilized with purified egg lecithin increased with increasing lecithin concentration. ${ }^{30}$ Formulation A5 (32.28 $\pm 2.74 \mathrm{cP})$ had higher viscosity when compared to formulation A1 (27.03 $\pm 3.60 \mathrm{cP})$ (Table 2). These observations were in agreement with the findings of Chanana and Sheth in which the increase in emulsion viscosity was found to be proportional to the amount of polyhydroxy alcohols (glycerin and propylene glycol) added to the formulation. ${ }^{26}$

\section{Stress sweep test}

Within the LVE region, formulations A1, A2, A4, and A5 showed that the value of $\mathrm{G}^{\prime}$ (storage modulus) was higher than $G^{\prime \prime}$ (loss modulus), which indicates that the systems showed more elastic behavior than viscous behavior (Figure 2). Loss modulus was higher than storage modulus for formulation A3, which indicates that the viscous properties dominated the elastic properties..$^{31}$ As the liquid oil content increased, the elastic properties were reduced and viscous properties were enhanced. These results indicate that an increase in liquid oil content in the formulation reduces elastic properties but enhances viscous properties. Table 2 shows that formulation $\mathrm{A} 2$ had a wider LVE region $(2.00 \mathrm{~Pa})$ when compared to A1 (0.32 Pa).

Test samples with high stability have a wide LVE region, whereas test samples with a weak and sensitive structure have a narrow LVE region. As the volume of lipid increased from $20 \%$ to $30 \%$, the width of the LVE region increased. This result is in agreement with the findings from Lippacher et al in which the increasing concentration of lipid leads to an increase in the particle-particle interactions and results in a more rigid structure. ${ }^{19}$ Formulations $\mathrm{A} 3$ and $\mathrm{A} 4$ had a narrower LVE region when compared to formulations A1, $\mathrm{A} 2$, and $\mathrm{A} 5$ (Table 2). This indicates that $\mathrm{A} 3$ and $\mathrm{A} 4$ broke down more easily with vibrations and small movements, and therefore A1, A2, and A5 were predicted to have a longer shelf life than A3 and A4. This result is in agreement with the result of accelerated stability testing, which found that formulations $\mathrm{A} 3$ and $\mathrm{A} 4$ were less stable when compared to formulations A1, A2, and A5.

\section{Accelerated stability testing}

The accelerated stability test is important for a cosmetic product to ensure its intended efficacy, physical, chemical, and microbiological quality is maintained over a period of time under certain conditions. A long-term stability study of a cosmetic product under "real life" conditions (room temperature $25^{\circ} \mathrm{C}$ ) is time consuming and labor intensive. Therefore, accelerated stability testing is an alternative for normal long-term stability studies, and can be carried out by subjecting a cosmetic product under elevated conditions over a period of time and observing the changes in physical appearance. The capability of cosmetic products to remain stable for 3-4 months of elevated temperature and three to four freeze-thaw cycles is considered as having an adequate shelf life. ${ }^{32}$

Formulations A1, A2, and A5 remained stable (no phase separation) under centrifugation condition, three freeze-thaw cycles, and 3-month storage at $25^{\circ} \mathrm{C}$ and $45^{\circ} \mathrm{C}$. Formulation A4 was found to separate into two layers in the first month at $45^{\circ} \mathrm{C}$, whereas formulation $\mathrm{A} 3$ separated in the second month. These observations are in agreement with the particle 

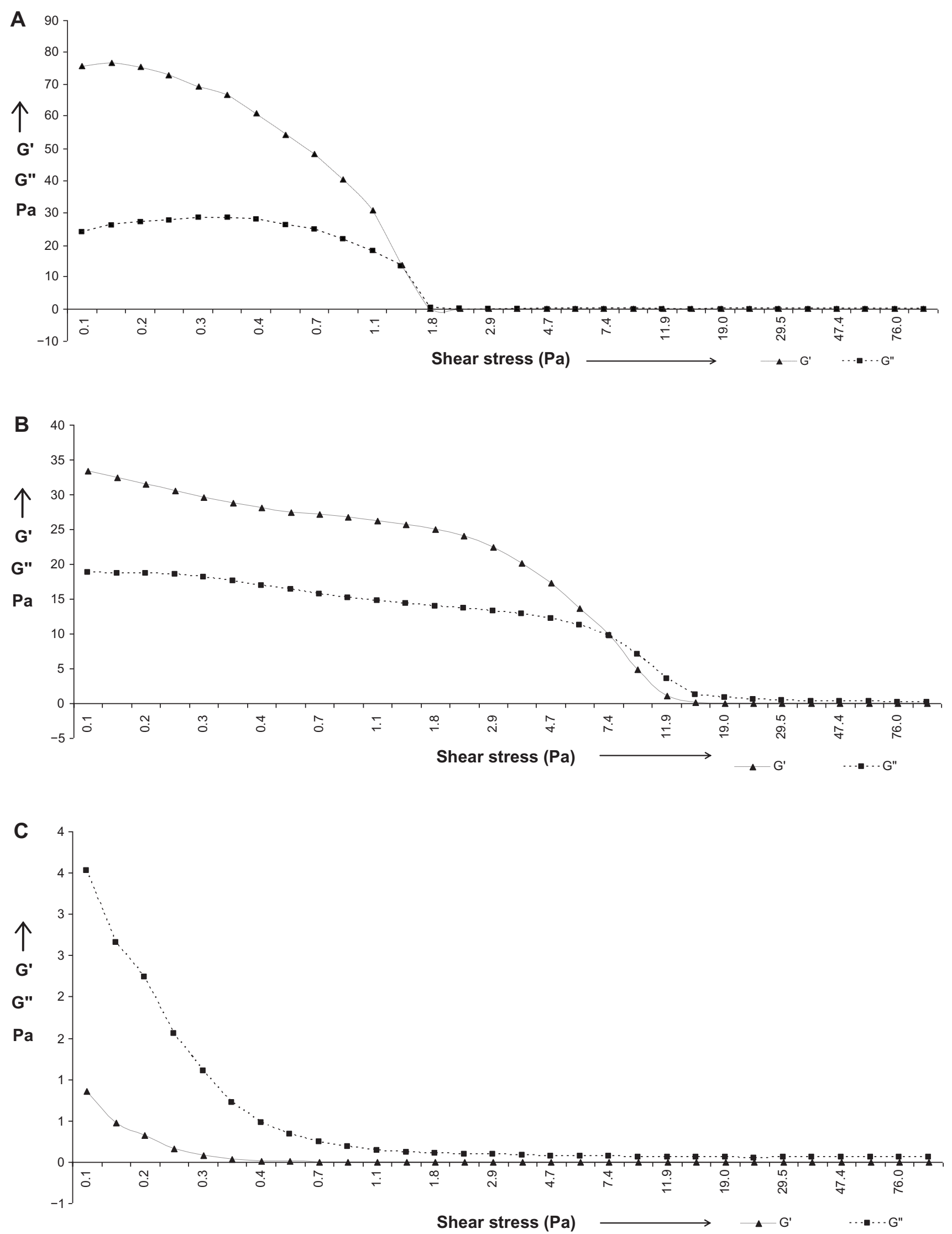

Figure 2 (Contined) 

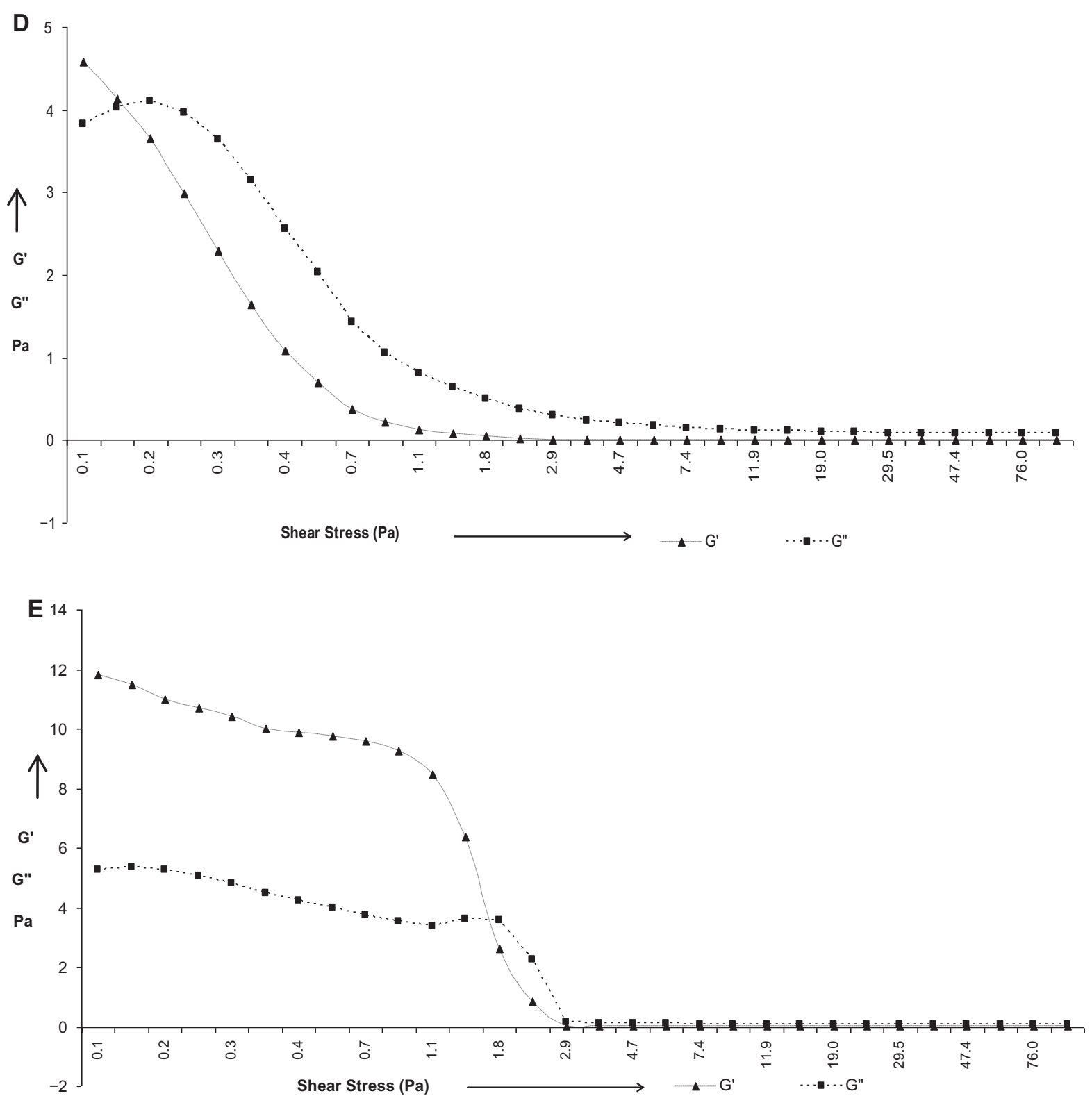

Figure 2 Stress sweep curve of the nanostructured lipid carrier formulations (A) AI, (B) A2, (C) A3, (D) A4, and (E) A5.

Abbreviations: AI, nanostructured lipid carrier with $20 \%$ lipids and a solid lipid/liquid lipid ratio of $90: 10$; A2, nanostructured lipid carrier with $30 \%$ lipids; A3, nanostructured lipid carrier with $20 \%$ lipids and a solid lipid/liquid lipid ratio of 50:50; A4, nanostructured lipid carrier with lecithin added; A5, nanostructured lipid carrier with propylene glycol added.

size study, which showed a sudden increase in particle size in the second and third months of storage. No gel formation was observed at $5^{\circ} \mathrm{C}$ for all the NLC formulations. Gel formation is not desirable as it may promote the expulsion of bioactives from NLC particles.

\section{Efficacy testing}

\section{Skin hydration}

Proper labeling is crucial if the cosmetic product is ready to be marketed. Clinical studies need to be carried out in order to provide evidence to support the claims on the label. The aim of the study was to investigate the effect of lipid content and presence of humectant on skin hydration and skin occlusion.

Application of NLC formulations (A1, A2, A3, A4, and A5) for 7 days led to a highly significant increase in average skin hydration from day one to day seven $(P<0.001)$, whereas untreated controls remained almost unchanged (30.65 arbitrary units on day one and 31.80 arbitrary units on day seven; $P>0.05$ ) (Figure 3). The application of NLCs led to 


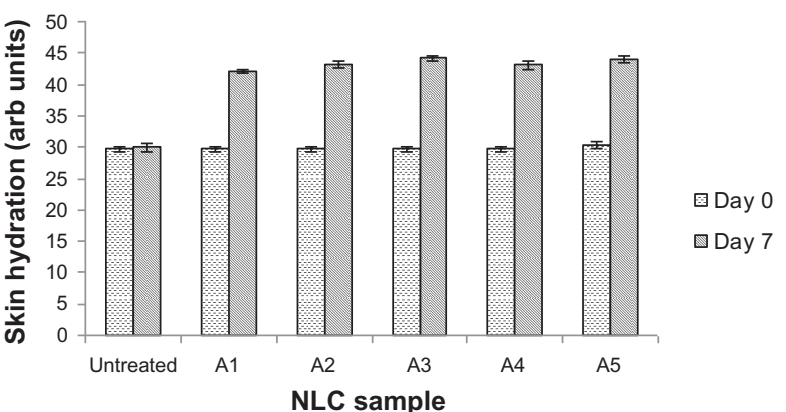

Figure 3 Skin hydration after the application of different nanostructured lipid carrier formulations for 7 days.

Abbreviations: AI, nanostructured lipid carrier with $20 \%$ lipids and a solid lipid/liquid lipid ratio of 90:10; A2, nanostructured lipid carrier with $30 \%$ lipids; A3, nanostructured lipid carrier with $20 \%$ lipids and a solid lipid/liquid lipid ratio of 50:50; A4, nanostructured lipid carrier with lecithin added; A5, nanostructured lipid carrier with propylene glycol added; Arb, arbitrary; NLC, nanostructured lipid carrier.

an increase in skin hydration due to the presence of occlusive agents found in the NLCs, ie, solid lipid (hydrogenated palm kernel glycerides) and liquid lipid (isopropyl palmitate). Due to the formation of an occlusive film on the skin, water evaporation from the skin was reduced for the area treated with NLCs. This result is in agreement with the finding by Pardeike and Muller in which NLC-containing cream increased the skin hydration more pronouncedly when compared to conventional cream and untreated controls. ${ }^{33}$

The NLC with $30 \%$ lipids (formulation A2) increased skin hydration more effectively when compared to the NLC with 20\% lipids (formulation A1) (Figure 3). This indicates that skin hydration was further enhanced by the high concentration of lipids. Wissing and Muller reported that skin occlusion is dependent on the sample volume, particle size, and the crystallinity of lipids. ${ }^{34}$ The occlusive agent found in NLCs (high concentration of lipid) was responsible for retaining moisture in the stratum corneum. This result is in agreement with the findings of Souto et al. ${ }^{35}$

After the 7-day application of the NLC containing lecithin (formulation A4) on the skin, the increase in skin hydration was comparable to the NLC without lecithin (formulation A1). Percentage increase in skin hydration was $49.09 \%$ and $42.62 \%$ for formulations A4 and A1, respectively (Figure 4). Treatment with formulation A4 increased skin hydration slightly higher than formulation A1, which could be due to the addition of occlusive agent (lecithin) to the formulation. The NLC containing propylene glycol (formulation A5) also showed comparable percentage increase in skin hydration when compared to the NLC formulation without propylene glycol (formulation A1). Percentage increase in skin hydration for formulations A5 and A1 were $48.04 \%$ and $42.62 \%$, respectively (Figure 4). Formulation A5 had slightly higher

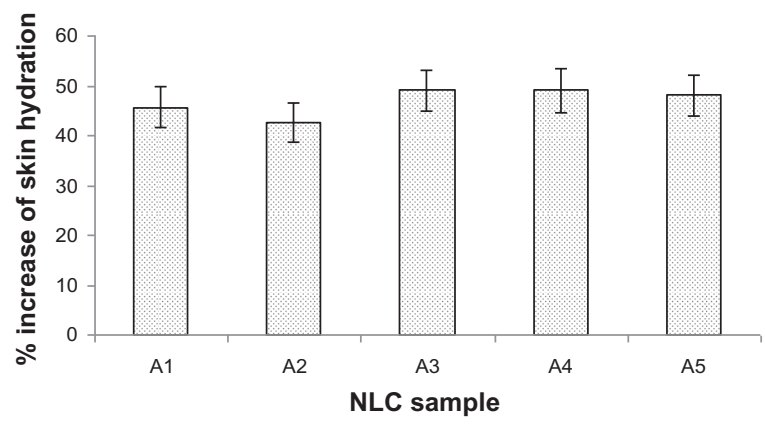

Figure 4 Percentage increase in skin hydration after the application of different nanostructured lipid carrier formulations for 7 days.

Abbreviations: AI, nanostructured lipid carrier with $20 \%$ lipids and a solid lipid/liquid lipid ratio of 90:10; A2, nanostructured lipid carrier with $30 \%$ lipids; $A 3$, nanostructured lipid carrier with $20 \%$ lipids and a solid lipid/liquid lipid ratio of 50:50; A4, nanostructured lipid carrier with lecithin added; A5, nanostructured lipid carrier with propylene glycol added; NLC, nanostructured lipid carrier.

percentage increase compared to formulation A1 due to the presence of humectant (propylene glycol), which increased the hydration of the stratum corneum by drawing water from the dermis and air. ${ }^{36}$

\section{Transepidermal water loss}

Bioactives penetration into the stratum corneum can be enhanced by occlusion caused by the product, which enhances hydration of the stratum corneum due to the inhibition of water evaporation. ${ }^{37}$ Average transepidermal water loss from the untreated test area remained the same (8.11 arbitrary units on day one and 8.48 arbitrary units on day seven; $P>0.05$ ) (Figure 5). Application of NLCs (formulation A1, A2, A3, $\mathrm{A} 4$, and $\mathrm{A} 5$ ) on the skin helped to reduce water loss from the skin when compared to the untreated control (Figure 5). This could be due to the small size of particles in NLCs having larger surface area, which give greater adhesive properties. ${ }^{38}$

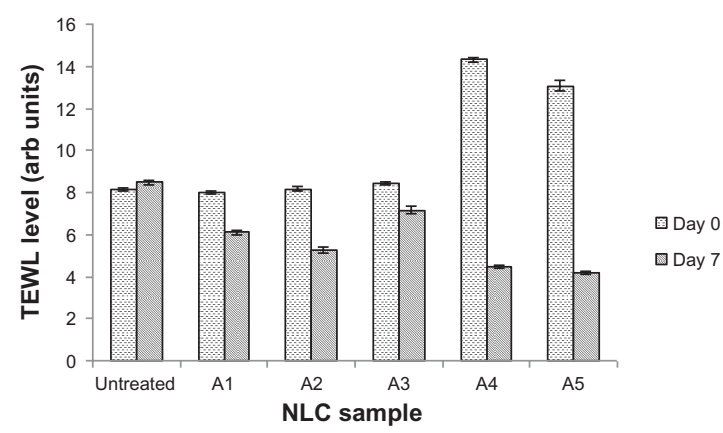

Figure 5 Transepidermal water loss after the application of different nanostructured lipid carrier formulations for 7 days.

Abbreviations: AI, nanostructured lipid carrier with $20 \%$ lipids and a solid lipid/ liquid lipid ratio of 90:10; A2, nanostructured lipid carrier with $30 \%$ lipids; A3, nanostructured lipid carrier with $20 \%$ lipids and a solid lipid/liquid lipid ratio of 50:50; A4, nanostructured lipid carrier with lecithin added; A5, nanostructured lipid carrier with propylene glycol added; Arb, arbitrary; NLC, nanostructured lipid carrier; TEWL, transepidermal water loss. 
They form a uniform compact layer on the skin surface, thus preventing water evaporation from the skin. ${ }^{39}$

The NLC containing 30\% lipids (formulation A2) was slightly more effective in reducing water loss from the stratum corneum compared to the NLC containing $20 \%$ lipids (formulation A1). Percentage reduction of transepidermal water loss for formulation A2 was $21.40 \%$, whereas it was $19.24 \%$ for formulation A1 (Figure 6). This result is in agreement with the skin hydration result in which skin occlusion is directly proportional to skin hydration, indicating that high occlusion gives high skin hydration. This result is also in agreement with the work done by Wissing and Muller in which skin occlusion is strongly dependent on the lipid concentration..$^{39}$ This could be due to the additional lipids absorbed into the intercellular space of the stratum corneum that help to reduce transepidermal water loss. ${ }^{7}$

The NLC with high solid lipid content (90:10; formulation A1) showed a much higher percentage reduction of water loss compared to the NLC with low solid lipid content (50:50; formulation A3). Percentage reduction of transepidermal water loss for A1 was $19.24 \%$, whereas it was $2.82 \%$ for formulation A3 (Figure 6). A similar finding was observed by Wissing and Muller in which the formulation in the presence of a highly crystalline lipid reduced water loss from the stratum corneum more effectively. ${ }^{39}$ Crystallinity in solid lipids was much higher than in liquid lipids, thus formulation A1 showed higher occlusivity compared to formulation A3.

The addition of lecithin into an NLC formulation effectively reduced water loss from the skin when compared to the untreated controls $(P<0.001)$. Percentage of reduction

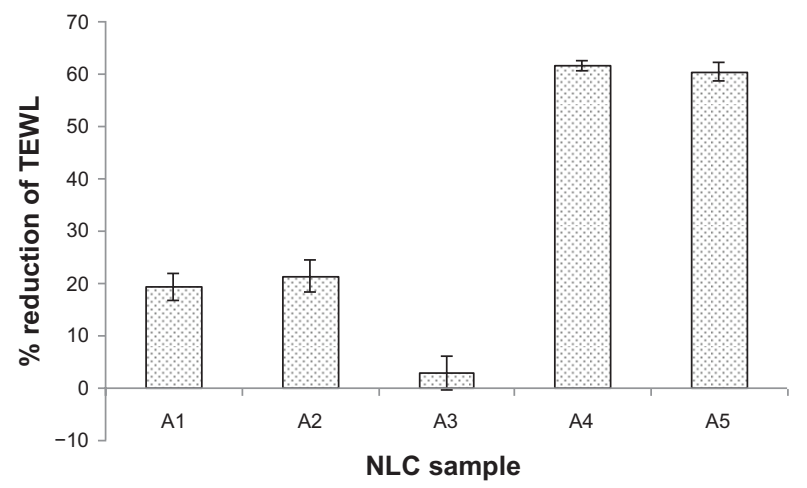

Figure 6 Percentage decrease in transepidermal water loss after the application of different nanostructured lipid carrier formulations for 7 days.

Abbreviations: AI, nanostructured lipid carrier with $20 \%$ lipids and a solid lipid/liquid lipid ratio of 90:10; A2, nanostructured lipid carrier with $30 \%$ lipids; A3, nanostructured lipid carrier with $20 \%$ lipids and a solid lipid/liquid lipid ratio of 50:50; A4, nanostructured lipid carrier with lecithin added; A5, nanostructured lipid carrier with propylene glycol added; NLC, nanostructured lipid carrier; TEWL, transepidermal water loss. of water loss for the NLC with lecithin (61.64\%; formulation A4) was much higher than the NLC without lecithin (19.24\%; formulation A1) (Figure 6). Lecithin is an emollient which helps to replace the absence of natural skin lipids in the space between corneocytes in the stratum corneum to prevent transepidermal water loss. ${ }^{40}$

After the 7-day application of the NLC containing propylene glycol (formulation A5), a highly significant difference in the reduction of transepidermal water loss as compared to the untreated controls was observed $(P<0.001)$. The addition of propylene glycol helped to reduce water loss from the skin dramatically when compared to the NLC without propylene glycol (formulation A1). Percentage reduction of transepidermal water loss for formulation A5 was $60.43 \%$, whereas it was $19.24 \%$ for formulation A1 (Figure 6). Propylene glycol is an odorless liquid which functions as a humectant and occlusive agent, and thus has the ability to form a film on the skin evenly and help to reduce the evaporation of water from the skin barrier. ${ }^{41}$

\section{Conclusion}

NLC formulations A1, A2, and A5 exhibited good physical stability in terms of particle size. The in vivo study showed that all the NLC formulations were able to significantly increase skin hydration in 7 days of treatment. All the NLC formulations reduced transepidermal water loss within 7 days and this effect was more pronounced in NLCs with the addition of propylene glycol or lecithin. In conclusion, NLCs with the addition of propylene glycol, lecithin, high lipid content, and solid lipid content are promising systems for cosmetics, with a proven effect on skin hydration and transepidermal water loss.

\section{Acknowledgments}

The authors would like to acknowledge the Malaysian Palm Oil Board, Graduate Students' Assistantship Scheme for the research grant. The authors would also like to thank the Malaysian Palm Oil Board and Universiti Putra Malaysia (Faculty of Science and the Institute of Bioscience) for the facilities used in the project.

\section{Disclosure}

The authors report no conflicts of interest in this work.

\section{References}

1. Potts RO, Francoeur ML. The influence of stratum corneum morphology on water permeability. J Invest Dermatol. 1991;96(4):495-499. 
2. Rougier A, Dupuis D, Lotte C, Roguet R, Schaefer H. In vivo correlation between stratum corneum reservoir function and percutaneous absorption. J Invest Dermatol. 1983;81(3):275-278.

3. Eckert RL, Rorke EA. Molecular biology of keratinocyte differentiation. Environ Health Perspect. 1989;80:109-116.

4. Harada K, Murakami T, Yata N, Yamamoto S. Role of intercellular lipids in stratum corneum in the percutaneous permeation of drugs. J Invest Dermatol. 1992;99(3):278-282.

5. Lavrijsen AP, Bouwstra JA, Gooris GS, Weerheim A, Bodde HE, Ponec M. Reduced skin barrier function parallels abnormal stratum corneum lipid organization in patients with lamellar ichthyosis. J Invest Dermatol. 1995;105(4):619-624.

6. Cua AB, Wilhelm KP, Maibach HI. Elastic properties of human skin: relation to age, sex, and anatomical region. Arch Dermatol Res. 1990; 282(5):283-288.

7. Elsner P, Wilhelm D, Maibach HI. Mechanical properties of human forearm and vulvar skin. Br J Dermatol. 1990;122(5):607-614.

8. Vogel HG. Directional variations of mechanical parameters in rat skin depending on maturation and age. $J$ Invest Dermatol. 1981;76(6):493-497.

9. Riviere JE, Papich MG. Veterinary Pharmacology and Therapeutics. 9th ed. Ames, IA: John Wiley and Sons; 2009.

10. Leveque JL, de Rigal J. In vivo measurement of the stratum corneum elasticity. Bioeng Skin. 1985;1:13-23.

11. Elsner P, Maibach HI, editors. Cosmeceuticals: Drugs vs Cosmetics. New York, NY: Marcel Dekker; 2000:23-24.

12. Wissing SA, Muller RH. The influence of solid lipid nanoparticles on skin hydration and viscoelasticity - in vivo study. Eur J Pharm Biopharm. 2003;56(1):67-72.

13. Hentschel A, Gramdorf S, Muller RH, Kurz T. $\beta$-carotene-loaded nanostructured lipid carriers. J Food Sci. 2008;73(2):N1-N6.

14. Muller RH, Peterson RD, Hommoss A, Pardeike J. Nanostructured lipid carriers (NLC) in cosmetic dermal products. Adv Drug Delivery Rev. 2007;59(6):522-530.

15. Hung LC, Basri M, Tejo BA, et al. An improved method for the preparations of nanostructured lipid carriers containing heat-sensitive bioactives. Colloids Surf B Biointerfaces. 2011;87(1):180-186.

16. Heimenz PC. Principles of Colloid and Surface Chemistry. New York, NY: Marcel Dekker; 1986.

17. Mitri K, Shegokar R, Gohla S, Anselmi C, Muller RH. Lipid nanocarriers for dermal delivery of lutein: preparation, characterization, stability and performance. Int J Pharm. 2011;414(1-2):267-275.

18. Lim HN, Kassim A, Huang NM, Yarmo A, Yeong SK. Study of highly concentrated olive oil-in-water emulsions stabilized by palm-based nonionic surfactant. Sains Malays. 2009;38(1):95-102.

19. Lippacher A, Muller RH, Mader K. Liquid and semisolid SLN dispersions for topical application: rheological characterization. Eur J Pharm Biopharm. 2004;58(3):561-567.

20. Bais D, Trevisan A, Lapasin R, Partal P, Gallegos C. Rheological characterization of polysaccharide-surfactant matrices for cosmetic o/w emulsions. J Colloid Interface Sci. 2005;290(2):546-556.

21. Teeranachaideekul V, Souto EB, Junyaprasert VB, Muller RH. Cetyl palmitate-based NLC for topical delivery of coenzyme Q10 - development, physicochemical characterization and in vitro release studies. Eur J Pharm Biopharm. 2007;67(1):141-148.
22. Phipps LW. The High Pressure Dairy Homogenizer. Ithaca, NY: Cornell University; 2009:44-78.

23. Seetapan N, Bejrapha P, Srinuanchai W, Ruktanonchai UR. Rheological and morphological characterizations on physical stability of $\gamma$-oryzanolloaded solid lipid nanoparticles (SLNs). Micron. 2010;41(1):51-58.

24. Benita S, editor. Microencapsulation: Methods and Industrial Applications. New York, NY: Marcel Dekker; 1996:213-216.

25. Beck R, Guterres S, Pohlmann A, editors. Nanocosmetics and Nanomedicines: New Approaches for Skin Care. Berlin: SpringerVerlag; 2011:163-169.

26. Chanana GD, Sheth BB. Particle size reduction of emulsions by formulation design. I: effect of polyhydroxy alcohols. J Parenter Sci Technol. 1993;47(3):130-134

27. Han F, Li S, Yin R, Liu H, Xu L. Effect of surfactants on the formation and characterization of a new type of colloidal drug delivery system: nanostructured lipid carriers. Colloids Surf A Physiochem Eng Asp. 2008;315(1-3):210-216.

28. Floyd AG. Top ten considerations in the development of parental emulsions. Pharm Sci Technol Today. 1999;2(4):134-143.

29. Freitas C, Muller RH. Correlation between long-term stability of solid lipid nanoparticles (SLN) and crystallinity of the lipid phase. Eur $J$ Pharm Biopharm. 1999;47(2):125-132.

30. Ishii F, Sasaki I, Ogata H. Effect of phospholipid emulsifiers on physicochemical properties of intravenous fat emulsions and/or drug carrier emulsions. J Pharm Pharmacol. 1990;42(7):513-515.

31. Junyaprasert VB, Teeranachaideekul V, Souto EB, Boonme P, Muller RH. Q10-loaded NLC versus nanoemulsions: stability, rheology and in vitro skin permeation. Int J Pharm. 2009;377(1-2):207-214

32. Barel AO, Paye M, Maibach HI. Handbook of cosmetic science and technology. Marcel Dekker, New York. 2001:769-781.

33. Pardeike J, Muller RH. In vivo skin hydration properties of a coenzyme Q10 containing cream with nanostructured lipid carriers (NLC) [abstract]. AAPS J. 2006;8(Suppl 2):001660.

34. Wissing SA, Muller RH. A novel sunscreen system based on tocopherol acetate incorporated into solid lipid nanoparticles. Int J Cosmet Sci. 2001;23(4):233-243.

35. Souto EB, Wissing SA, Barbosa CM, Muller RH. Development of a controlled release formulation based on SLN and NLC for topical clotrimazole delivery. Int J Pharm. 2004;278(1):71-77.

36. Prendergast PM, Shiffman MA, editors. Aesthetic Medicine: Art and Techniques. Berlin: Springer-Verlag; 2011:77.

37. Jenning V, Gysler A, Schafer-Korting M, Gohla SH. Vitamin A loaded solid lipid nanoparticles for topical use: occlusive properties and drug targeting to the upper skin. Eur J Pharm Biopharm. 2000;49(3): 211-218.

38. Tripathi G, Dubey BK. In vitro occlusion studies of solid lipid nanoparticles loaded hydrogel by water vapour permeability analysis. JGPT. 2011;3(4):14-20.

39. Wissing SA, Muller RH. The influence of the crystallinity of lipid nanoparticles on their occlusive properties. Int J Pharm. 2002; 242(1-2):377-379

40. Draelos ZD, editor. Cosmetic Dermatology: Products and Procedures. Oxford: John Wiley and Sons; 2010:126-127.

41. Farage MA, Miller KW, Maibach HI, editors. Textbook of Aging Skin. Berlin: Springer-Verlag; 2010:933.

International Journal of Nanomedicine

\section{Publish your work in this journal}

The International Journal of Nanomedicine is an international, peerreviewed journal focusing on the application of nanotechnology in diagnostics, therapeutics, and drug delivery systems throughout the biomedical field. This journal is indexed on PubMed Central, MedLine, CAS, SciSearch $\AA$, Current Contents ${ }^{\circledR} /$ Clinical Medicine,

Journal Citation Reports/Science Edition, EMBase, Scopus and the Elsevier Bibliographic databases. The manuscript management system is completely online and includes a very quick and fair peer-review system, which is all easy to use. Visit http://www.dovepress.com/ testimonials.php to read real quotes from published authors. 